\title{
Sisters find a slick way to stick
}

In some ways, meiotic division is like country dancing. During meiosis I, homologous chromosome pairs line up opposite one another and then, at anaphase I, whirl apart to opposite poles of the cell. But in the next reel, meiosis II, the sister chromatids themselves separate. So what is the molecular 'caller' that tells the partners when to stick together and when to separate? Dean Dawson and colleagues, reporting in October's Current Biology, have identified a key player in the process.

Clues to what's going on in meiosis can be gleaned from events during mitosis. Sister chromatids are initially bound along their length by a physical tie - the so-called cohesin complex. Consisting of four subunits, Smclp, Smc3p, Scclp and Scc3p, cohesin establishes this connection and maintains it until anaphase. At this point, sister-chromatid cohesion is lost - first along the arms and then at the centromeres by proteolytic cleavage of the Scclp subunit. This cohesion must be maintained during meiosis I and, in addition, the kinetochores (multiprotein complexes at the centromeres) must be regulated such that both sisters are pulled to one pole rather than being dragged away from each other.

Dawson and colleagues realized that yeast with a mutation in the SLK19 (synthetic lethal kar3) gene show a defect in meiosis consistent with a failure in the control of sister-chromatid behaviour: rather than forming tetrads containing four haploid spores, these mutants form dyads with two diploid spores. The authors found that although slk19 mutants enter meiosis I efficiently, the sister chromatids then separate to opposite poles of the cell. Moreover, most of the mutants do not go through a second meiotic division - that's why they form dyads. This could be due to a breakdown of sister-chromatid cohesion, to impaired kinetochore function, or to both.

Dawson and co-workers next used indirect immunofluorescence to monitor the localization of Slk19p during meiosis. The picture shows synaptonemal complexes (red; these run along paired meiotic chromosomes) and Slk19p-GFP (green). The authors found that Slk19p localizes to centromeric regions during prophase I, and that it remains there until anaphase I. After this point, however, Slk19p loses its association with the centromeres, and localizes instead along the spindles, as it is left behind when the centromeres migrate polewards at meiosis I.

These localization patterns indicate that
Slk19p might keep the sister chromatids together during the first meiotic division. But how? The authors speculated that it could affect Rec8p, which is the yeast meiosis-specific homologue of Scclp. Rec8p must be localized at the centromeres to ensure sister-chromatid cohesion through meiosis I, so perhaps Slk19p prevents the degradation of Rec8p? To test this, Dawson and colleagues looked at the effect of Slk19p on localization of Rec8p. They found that, during the early stages of meiosis I, the localization of Rec8p is indistinguishable between slk19 and wild-type strains. In anaphase I, however, Rec8p is maintained at the centromeres in $80 \%$ of the wild-type cells, but in most of the $s l k 19$ cells there is almost no Rec8p staining.

How can a protein found in mitotic cells specifically regulate the behaviour of sister chromatids during meiosis? The answer may be that Slk19p acts downstream of another meiosis-specific factor, and the authors' top candidate is a putative meiotic regulatory protein, Spo13p. Both spo13 and slk19 mutants have defects in the behaviour of sister chromatids during meiosis. But the defect in spo13slk19 double mutants is no more serious than that in the spo13 mutant alone, indicating that the two proteins may be involved in a common pathway. The next step in studying the molecular dance of meiosis is to work out how Slk19p and Spo13p might prevent the partners from separating too soon.

\section{(2) References and links}

Alison Mitchell ORIGINAL RESEARCH PAPER Kamieniecki, R. J., Shanks, R. M. Q. \& Dawson, D. S. Slk19p is necessary to prevent separation of sister chromatids. Curr. Biol. 10, 1182-1190 (2000) FURTHER READING Nasmyth, K., Peters, J.-M. \& Uhlmann, F. Splitting the chromosome: cutting the ties that bind sister chromatids. Science 288, 1379-1384 (2000)

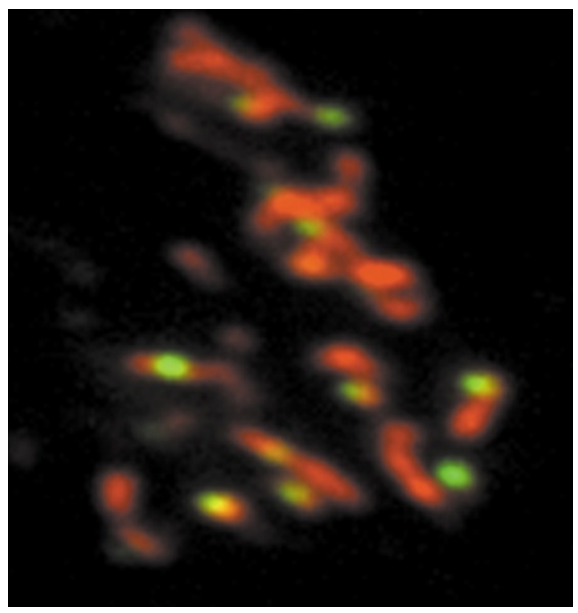

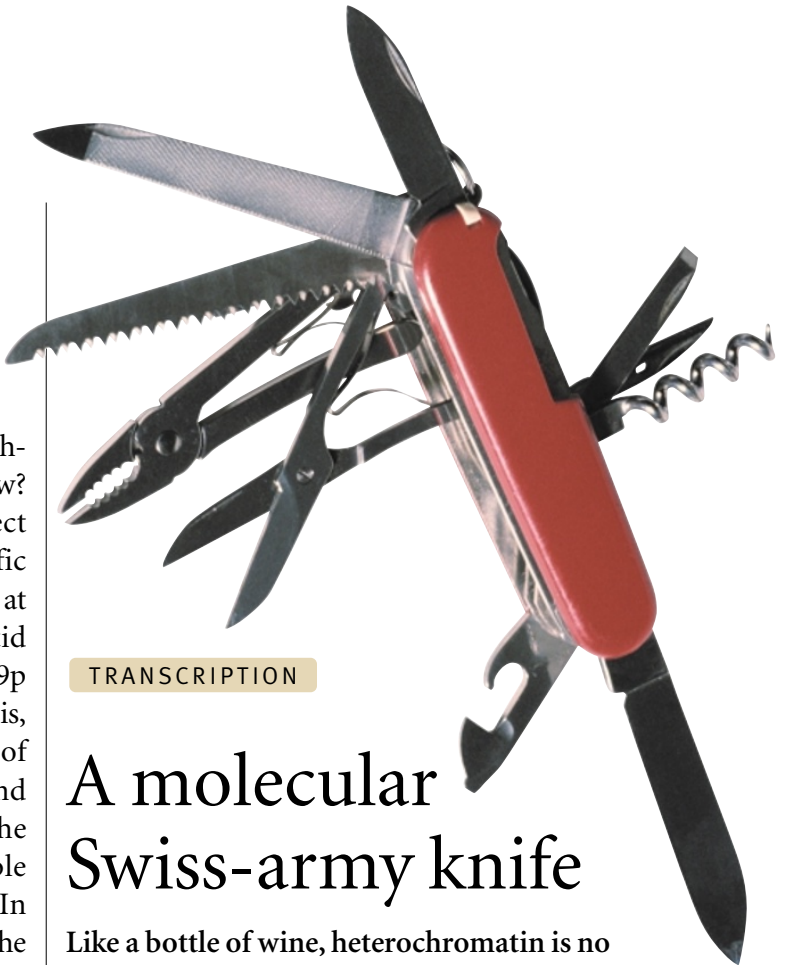

use unless you can open it. TAF250 is one protein that helps to uncork the DNA by loosening histone H1's grip on it. A paper by Pham and Sauer in Science suggests that TAF250 is more a Swiss-army knife than a corkscrew, though: it was already known to phosphorylate and acetylate histone $\mathrm{H} 1$, but now it adds ubiquitylation to its list of talents list.

Polyubiquitylation — adding a chain of ubiquitin molecules to proteins - is a signal for destruction, but monoubiquitylation is less well understood. Knowing that histone $\mathrm{H} 1$ can be monoubiquitylated, Pham and Sauer set out to discover what was doing it, and found TAF250. Ubiquitylation requires a minimum of two enzyme activities, an activator and a conjugator, which are usually on separate proteins. In vitro assays revealed that TAF250 has both. But what about in vivo? Having identified the region of TAF250 that's necessary for histone monoubiquitylation, they created mutants that lack the activity and are heterozygous for dorsal - a maternally expressed gene important for early Drosophila development. In these mutants, expression of the dorsalresponse genes twist and snail was significantly reduced, resulting in a twisted phenotype. Levels of monoubiquitylated histone were also reduced. More substrates of TAF250's ubiquitylating activity, as well as factors that ubiquitylate the other histones, may await discovery.

Cath Brooksbank

\section{(2) References and links}

ORIGINAL RESEARCH PAPER Pham, A.-D. \& Sauer, F.

Ubiquitin-activating/conjugating activity of TAF250, a mediator of activation of gene expression in Drosophila. Science $\mathbf{2 8 9}$, 2357-2360 (2000)

FURTHER READING Mizzen, C. A. \& Allis, C. D.

New insights into an old modification. Science 289, 2290-2291 (2000) 